\title{
Polio outbreak in Nigeria linked to trivalent oral vaccine
}

Hopes that poliomyelitis might be confined to the annals of medical history have once again been dashed with the recent announcement that Nigeria is currently experiencing a large outbreak of the disease. In an ironic twist, the source of this latest outbreak is the trivalent oral polio vaccine (OPV), suggesting that the virus remains two steps ahead when it comes to outsmarting the determined efforts to eliminate it once and for all.

The Global Polio Eradication Initiative, commenced in 1988, vowed to eradicate poliomyelitis by targeting disease transmission and putting in place effective surveillance tactics to monitor the course of the wild-type virus. The Initiative has had undoubted success, largely due to the highly efficacious OPV; the number of cases of polio was reduced from 350,000 in 1988 , to 1998 in 2006. Today, polio remains endemic in only four countries: India, Pakistan, Afghanistan and Nigeria - the country at the heart of the current crisis. In the 28th September issue of Morbidity and Mortality weekly, the results of an extensive study that assessed the levels of poliomyelitis infections between January 2006 and August 2007 are presented. By far the most worrying aspect of this report are the 69 confirmed cases of poliomediated paralysis in Northern Nigerian states reported between January 1st 2006 and August 17th 2007, all caused by vaccine derived poliovirus (VDPV). OPV consists of live-attenuated versions of all three poliovirus serotypes, is of clear benefit, but has been notoriously associated with problems in areas where vaccine take-up is low. Such circumstances are ripe for sustained polio circulation should the rare mutation events that confer a regain of virulence on the attenuated virus occur, all of which are promoted by, and exacerbated in, immunosuppressed populations.
The Nigerian outbreak was confirmed as having originated from the OPV when officials at the CDC noted that the type- 2 serotype was prevalent in samples arriving for analysis. Olen Kew of the CDC noted, "that instantly raised suspicions because wild-type 2 poliovirus has been eradicated globally". The inevitable conclusion that the only possible source of the Nigerian cases was the vaccine itself was confirmed with sequencing. It is likely that the scale of this most recent outbreak will continue to grow, certainly if history is anything to go by. Nigeria suffered a similar polio outbreak in 2003-2004 when antivaccine, anti-Western sentiments led to the cessation of vaccination efforts in a number of states, the disastrous consequences of which manifest themselves rapidly; by the end of 2004 the number of cases of polio reached 800, having already doubled, and by 2006 topped 1100. Moreover, wild-type poliovirus then reinfected 20 other countries, which had previously been free of the disease - putting a major dent in the Global Eradication Initative.

There has been criticism of the WHO and Nigerian health officials over the latest outbreak, with many claiming that the delay in the release of essential information might be highly damaging to the vaccination efforts. "There were legitimate concerns that anti-polio vaccination rumors would be rekindled by an incomplete explanation of the cause of the VDPV outbreak" warns Kew. It is essential that the need to vaccinate be reinforced, now more than ever. Further polio experts also expressed their concern at the apparent oversight by the WHO, highlighting the need for total transparency on the sources of all VDPV outbreaks and warning against any actions that might be misconstrued as being secretive. An open exchange of all available information on such outbreaks is essential if the nature and risk factors associated with VDPVs are to be evaluated and vaccine take-up rates are not to be adversely effected. The recent call for more rapid and sensitive RT-PCR-based assays to detect and distinguish between VDPVs has been addressed and the resulting up-to-the-minute assays are currently being tested. These tests may well aid the diagnostics of such infections but they will do little to help allay fears of the Nigerian people regarding vaccine safety in the face of this latest crisis.

\section{Sources : Science magazine}

www.sciencemag.org; and Morbidity and Mortality Weekly 56(38), 996-1001 (2007).

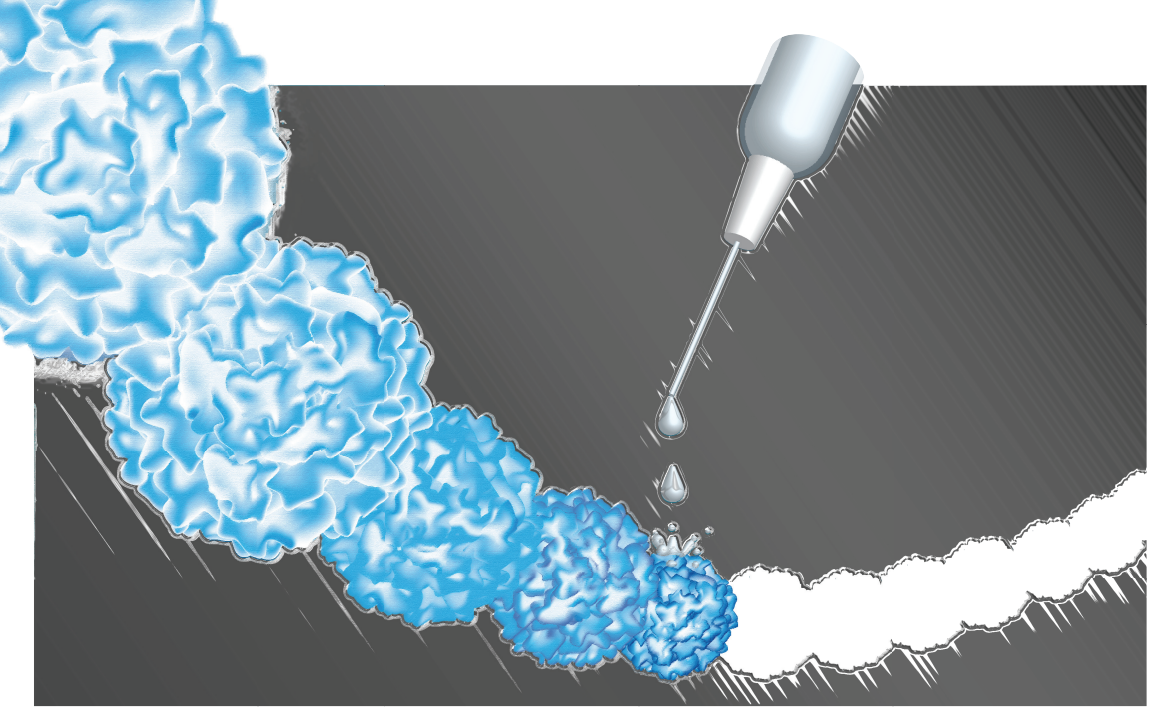




\section{Priority Paper Alerts}

GCV modulates the antitumoural efficacy of a replicative adenovirus expressing the TAT8-TK as a late gene in a pancreatic tumour model.

Cascante A, Abate-Daga D,

Garcia-Rodriguez L et al.: Gene Therapy 14, 1471-1480 (2007).

A study that addresses some of the controversy in the literature surrounding the effectiveness of antitumor adenovirus-mediated virotherapy. In this investigation the antitumor properties of an adenoviral vector expressing the herpes simplex thymidine kinase gene (Tat-TK), under control of a major late promoter (AdRGD-Tat8-TK-L) were examined when introduced in combination with ganciclovir (GCV) into pancreatic cancer cell lines. In three out of the four cell lines tested, GCV significantly enhanced the effectiveness of virotherapy, whilst correspondingly decreasing viral replication. The research presented uncovers the importance of scheduling in obtaining maximum benefits associated with AdRGDTat8-TK-L/GCV combination therapy, suggesting there is a role for such combination therapy provided that regimens incorporate two cycles of adenoviral application plus GCV.

A single mutation in the PB1-F2 of H5N1 (HK/97) and 1918 influenza A viruses contributes to increased virulence.

Conenello GM, Zamarin D, Perrone LA et al.: PLoS Pathogens 3(10), E141 (2007).

Pinning down the genetic roots of the enhanced pathogenicities of more virulent influenza strains is of high priority. Of particular relevance are possible sequence oddities of the more pathogenic strains, such as the 1918 'Spanish flu' influenza strain and the H5N1 strains associated with human infections. The proapoptotic PB1-F2 protein of influenza A has sparked recent interest owing to its capacity to contribute to pathogenicity in a mouse model. This report links a single amino acid change found at position 66 (N66S) of the PB1-F2 protein in the $1997 \mathrm{H} 5 \mathrm{~N} 1$ outbreak strain and the 1918 pandemic causing virus. The researchers demonstrate that in the mouse model, a recombinant virus expressing the N66S change possessed enhanced pathogenicity. Moreover, genetic modification of the 1918 strain to incorporate the revertant S66N change, resulted in a virus with a 3 log lower LD50, causing less morbidity and mortality than the wildtype virus. Both strains containing serine also resulted in enhanced cytokine production in lungs following infection.

\section{New comprehensive phylogenic analysis sheds light on the evolution of variola virus}

Although smallpox was successfully eradicated in 1980, the puzzle surrounding the evolution of this catastrophic human disease has long bemused researchers and sparked intense debate. Some of the questions surrounding the genetic origins of variola virus (VARV), the etiologic agent of smallpox, and the evolutionary timescale over which it has been active are addressed in a recent paper published in PNAS by groups from the CDC, GA and the Lawrence Livermore National Laboratory, CA, USA.

With the tell-tale signs of smallpoxlike skin rashes observed on Egyptian mummies dating from 1100 to 1580 B.C., the rest of medical history is correspondingly littered with similar, unsubstantiated claims of smallpoxlike disease rearing its ugly head across the globe. The earliest unmistakeable descriptions of smallpox are to be found in 4th century AD China, 7th century $\mathrm{AD}$ India and later, in 10th century AD southwestern Asia.

Waters were further muddied with the incontestable evidence that historical variola-like infections were derived from biologically distinct viral subtypes and hence, took correspondingly variable clinical courses. The most severe diseasecausing form, VARV-major, had an associated severe morbidity and mortality (more than 30\%). VARVmajor spread rapidly to populations of the American and sub-Saharan African continents with the push of the Western world to colonize these areas in the 15th century. By contrast, alastrim minor, a significantly less virulent form of VARV, was described as causing only mild disease in Central America and South Africa, in the 19th century. The historical evidence was apparently conflicting in some respects, did the presence of alastrim minor correspond to late VARV evolution or was divergence an ancient process?

'...the traditional nomenclature
separating VARV into major and
minor variants is inadequate...'

With an extensive analysis of concatenated single-nucleotide polymorphisms from the genome sequences of 47 geographically diverse VARV isolates, and combining this molecular information with historical records, researchers from the CDC were able to generate an in depth analysis of VARV evolution. In the resulting complex phylogeny, the authors describe the presence of two primary clades, formed upon divergence of a common ancestral VARV. They also identify the most likely source of this early ancestral VARV as being a rodent-borne African variola-like virus. The divergence of the two primary clades is demonstrated to have occurred 16,000-68,000 years before present (YBP). They also suggest that the traditional nomenclature separating VARV into major and minor variants is inadequate, however, on a gross level they demonstrate that the one primary clade, including the major disease-causing strains, is likely to have spread from Asia 400-1600 YBP with the remaining primary clade having diverged at 1400-6300 YBP. The results of this study provide a valuable insight in the origins of this devastating human pathogen and suggest that given the earlier divergence of VARV, the evolutionary time-frame over which VARV origins are considered requires further investigation.

Source: Li S, Carroll DS, Gardener SN et al:: On the origin of smallpox; correlating variola phylogenics with historical smallpox records. Proc. Natl Acad. Sci. USA 104, 15787-15792 (2007). 


\section{Ebola outbreak confirmed in the Democratic Republic of Congo}

The WHO has activated its Global Outbreak Alert and Response Network as cases of Ebola Hemorrhagic fever have now been confirmed in the Democratic Republic of Congo (DMC). The headline-grabbing virus, notorious for the sporadic nature of its outbreaks, has emerged in the southern DMC province of Kasai-Occidental; the current death toll stands at 171 . However, warns Gregory Hartl, a WHO spokesman, "not all these deaths are necessarily due to Ebola". The WHO and CDC teams, deployed at the request of the DMC Ministry of Health, face some challenges in distinguishing true Ebola cases from those of other diseases rife in the area at this time, given that apparent mortality rates in this current outbreak seem uncharacteristically low for the Ebola virus and reported symptoms are confused. Early diagnostics indicate some patients as being infected with Shigella dysenteriae and Salmonella typhi. "We have to identify and isolate cases, separating Shigella patients from Ebola," Hartl stresses.

The Ebola virus has been confirmed in a number of patients and the CDC and WHO teams must now continue with their efforts to track the course of the virus - a process somewhat complicated by the remoteness of the region involved in the outbreak and worse still, by the ongoing conflict in other parts of the country. Ebola, a Filovirus, caused a similar outbreak in the DMC (formerly Zaire) in 1995 , in which 233 people died. Ebola is famed for its high case-mortality rate, $50-90 \%$ and its accompanying distressing symptoms. There is no known specific treatment or prophylactic measure, with doctors having to rely on supportive therapies to manage patients. The source of the virus, and the reasons behind the sporadic nature of its outbreaks, had remained elusive until very recently, when it was suggested that the natural reservoir for the virus is fruit bats. The latest evidence suggests that an effective vaccine may be further away on the horizon than previously anticipated; conclusions drawn from a genetic study that highlights the remarkable genetic diversity of the Ebola virus, in part due to previously undemonstrated recombination processes occurring between distinct viral strains.

Source: New Scientist

www.newscientist.com

\section{Influenza immunization for all healthcare workers in the USA}

The problematic transmission of influenza from healthcare worker to patient in the clinical setting is being targeted by the American College of Physicians (ACP). The ACP is now recommending an annual influenza vaccine for all healthcare workers who come into direct contact with patients on a regular basis. It is encouraging all healthcare organizations to establish an annual influenza immunization program in an effort to reduce patient morbidity and mortality and nationwide expenditure on the disease. Influenza represents a serious public health threat, with some $20 \%$ of the US population becoming infected on an annual basis, an estimated 36,000 of these infections being fatal. The ACP's recent call for healthcare worker immunization is bolstered by well-documented cases of healthcare worker-patient transmissions.

With only $36 \%$ of such workers being immunized each year and an estimated $70 \%$ remaining at work despite suffering from influenza it seems only prudent to reinforce the requirement for vaccination. "Immunizing healthcare workers safely and effectively prevents a significant number of influenza infections, hospitalizations and deaths among the patients they care for, as well as preventing workplace disruption and medical errors by workers absent from work due to illness, or present at work but ill," says Vincenza Snow, MD, FACP, Director, Clinical Programs and Quality of Care at ACP.
Many major healthcare organizations have already endorsed the policies that stipulate their workers be vaccinated. Workers are exempt from the ACP's new policy on medical grounds, for reasons of religious objection and if they provide a signed statement of refusal. The ACP now goes further by asking such organizations to actively promote the influenza vaccine to their workers and to educate them to the benefits of such programs. It is hoped that this new initiative will also provide invaluable insights into the reasons for vaccine refusal, enabling steps to be implemented that may enhance vaccine take up rates in the future.

Source: www.sciencedaily.com

\section{About the Bulletin Board}

The Bulletin Board highlights some of the most important events and research in the field of virology. If you have newsworthy information, please contact: 